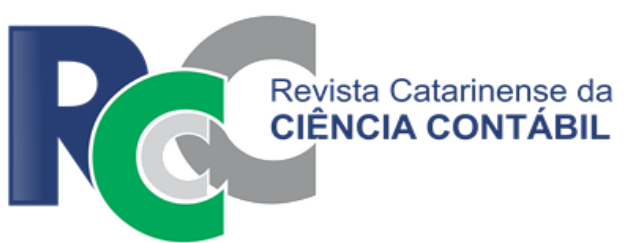

\title{
INDICADORES DE ENDIVIDAMENTO: ANÁLISE FATORIAL NA COMPARAÇÃO ENTRE A LITERATURA E A AGÊNCIA NACIONAL DE ENERGIA ELÉTRICA (ANEEL)
}

\author{
INDEBTEDNESS INDICATORS: FACTOR ANALYSIS IN THE COMPARISON \\ BETWEEN THE LITERATURE AND THE NATIONAL ELECTRIC ENERGY \\ $A G E N C Y$ (ANEEL)
}

\author{
ARACÉLI CRISTINA DE S. FERREIRA \\ Universidade Federal do Rio de Janeiro. Endereço: Laboratório de \\ Modelagem de Sistemas Contábeis | Av. Pasteur, 250, sala 247| Urca| 22290- \\ $240 \mid$ Rio de Janeiro/RJ | Brasil. \\ (1) http://orcid.org/0000-0003-3135-4664 \\ araceli@facc.ufrj.br
}

\section{DILO SERGIO DE CARVALHO VIANNA}

Universidade Federal do Rio de Janeiro. Endereço: Laboratório de Modelagem de Sistemas Contábeis | Av. Pasteur, 250, sala 247| Urca | 22290$240 \mid$ Rio de Janeiro/RJ | Brasil.

(D) http://orcid.org/0000-0003-1069-1928

dilo@facc.ufrj.br

\section{JAZMIN FIGARI DE LA CUEVA}

Universidade Federal do Rio de Janeiro. Endereço: Laboratório de Modelagem de Sistemas Contábeis | Av. Pasteur, 250, sala 247| Urca|22290$240 \mid$ Rio de Janeiro/RJ | Brasil.

(D) http://orcid.org/0000-0002-4282-9592

jazminfigari@gmail.com

\section{ANDRÉ LUIZ BUFONI}

Universidade Federal do Rio de Janeiro. Endereço: Laboratório de Modelagem de Sistemas Contábeis | Av. Pasteur, 250, sala 247| Urca|22290$240 \mid$ Rio de Janeiro/RJ | Brasil.

Dhttp://orcid.org/0000-0002-3568-0590

bufoni@facc.ufrj.br

\section{JULIANA MOLINA QUEIROZ}

Universidade Federal do Rio de Janeiro. Endereço: Laboratório de Modelagem de Sistemas Contábeis | Av. Pasteur, 250, sala 247| Urca| 22290$240 \mid$ Rio de Janeiro/RJ | Brasil.

(Dhttp://orcid.org/0000-0001-9398-2610

julianamolinaq@gmail.com

\section{RESUMO}

O objetivo deste artigo é verificar se o indicador de endividamento proposto pela Agência Nacional de Energia Elétrica (ANEEL) apresenta uma relação latente com os indicadores de endividamento utilizados pela literatura. A ANEEL sistematizou a supervisão econômico-financeira das 
operadoras de distribuição de energia por meio de indicadores de desempenho, entre eles o endividamento, o qual se diferencia dos utilizados na literatura. Neste estudo, o indicador da ANEEL de endividamento foi comparado com diferentes indicadores proxy de endividamento (Ribeiro \& Santos, 2004; Lima \& Freire, 2008; Ribeiro, Macedo, \& Marques, 2012; Delen, Kuzey, \& Uyar, 2013; Ozório, 2015; Castro et al., 2017). Para verificar se o indicador da ANEEL segue um padrão parecido com os demais, os indicadores foram agrupados por meio da análise fatorial. Os dados financeiros foram coletados nas demonstrações financeiras disponibilizadas pela ANEEL, no período de 2011 a 2018. Os resultados mostraram uma relação latente entre os indicadores de endividamento da ANEEL e três indicadores usados na literatura. É possível identificar que apesar de as distribuidoras e a ANEEL defenderem que a sistemática utilizada é setorial (ANEEL, 2014), o indicador de endividamento apresenta padrão de comportamento parecido com os utilizados pela literatura.

Palavras-chave: Indicadores. Endividamento. Setor elétrico. Análise fatorial.

\begin{abstract}
The objective of this article is to verify whether the indebtedness indicator proposed by the National Electric Energy Agency (ANEEL) presents a latent relationship with the indebtedness indicators used in the literature. ANEEL systematized the economic-financial supervision of energy distribution operators by means of performance indicators, among them the indebtedness, which differs from those used in literature. In this study, ANEEL's indebtedness indicator was compared with different proxy indebtedness indicators (Ribeiro \& Santos, 2004; Lima \& Freire, 2008; Ribeiro, Macedo, \& Marques, 2012; Delen, Kuzey, \& Uyar, 2013; Ozório, 2015; Castro et al., 2017). In order to verify that the ANEEL indicator follows a pattern similar to the others, the indicators were grouped by means of factor analysis. The financial data were collected in the financial statements made available by ANEEL, for the period from 2011 to 2018. The results showed a latent relationship between the ANEEL indebtedness indicators and three indicators used in the literature. It is possible to identify that although the distributors and ANEEL argue that the systematic used is sectoral (ANEEL, 2014), the indebtedness indicator presents a pattern of behavior similar to those used in the literature.
\end{abstract}

Keywords: Indicators. Indebtedness. Electricity sector. Factor analysis.

\title{
1 INTRODUÇÃO
}

O Setor Elétrico Brasileiro (SEB) é regulado pela Agência Nacional de Energia Elétrica (ANEEL). O SEB é composto majoritariamente por holdings, geradoras, transmissoras, comercializadoras e distribuidoras. Nos últimos anos, a ANEEL, por meio de notas técnicas, tem trabalhado com o intuito de promover o aprimoramento da supervisão econômico-financeira do SEB, particularmente do segmento de distribuição de energia e sua viabilidade financeira. Segundo Huenteler, Dobozi, Balabanyan e Banerjee (2017), a viabilidade financeira do setor de energia é um pré-requisito para atrair o investimento necessário para garantir o fornecimento confiável de energia e alcance universal à eletricidade. Além disso, a análise da viabilidade financeira serve como impulsionador da transição para a energia limpa (Huenteler et al. 2017).

Os modelos ou sistemáticas de supervisão do setor elétrico são compostos por dimensões de avaliação da viabilidade financeira (e.g.: financeira e operacional). A Nota Técnica 111/2016 apresentou a nova modelagem da ANEEL, a qual segue a lógica de que cada dimensão comporta um conjunto de indicadores de desempenho. Todavia, a necessidade de ajustes dinâmicos parece ser uma característica dos modelos de regulação. Essa necessidade de redefinição periódica dos parâmetros de regulação das operadoras de distribuição foi documentada por Ergas e Small (2001). 
No ano seguinte, Bakovic, Tenenbaum e Woolf (2002) apontaram que o design dos novos sistemas utilizados na regulação de alguns setores é fundamentalmente falho, segundo alguns investidores. Esta lista incluía, principalmente, as economias em desenvolvimento e em transição, incluindo o Brasil. Alguns anos depois, a Finlândia fez a primeira reforma do modelo regulatório de distribuição de eletricidade. Essa reforma ocorreu em 2012 e despertou o interesse pela reforma em outros países e em outros setores (por exemplo, gás e telecomunicações), além de um debate entre os profissionais e acadêmicos (Kuosmanen, Saastamoinen \& Sipilainen, 2013).

Em fevereiro de 2019, a British Columbia Hydro and Power Authority e a British Columbia Utilities Commission discutiram, entre outros assuntos, as oportunidades e os desafios associados à adoção da Regulação Baseada em Desempenho (PBR) (Lowry e Makos, 2020), indicando a necessidade de revisão de modelos regulatórios para focar na análise do desempenho. Ao longo dos anos, essa dinâmica vem provocando novos questionamentos dos modelos regulatórios e de seus indicadores de desempenho. Com isso, se mantém ativa a necessidade de trabalhos complementares. Nessa mesma linha de pensamento, Braga, Rufin, Brandão e Torres (2016) apontam a necessidade do estudo e do desenvolvimento dos modelos regulatórios ao indicar que “a supervisão financeira das empresas de distribuição de energia elétrica ainda está em estágio inicial em centros acadêmicos, em agências governamentais e entre reguladores internacionais".

A necessidade pela pesquisa a respeito dos modelos regulatórios também é documentada por Huenteler et al. (2017) ao sugerir que há uma limitação na pesquisa sobre os determinantes institucionais e os pré-requisitos de recuperação de custos e viabilidade financeira. Os autores também indicam que a pesquisa explicativa sobre a viabilidade financeira deve ser rigorosa, além de defenderem que a literatura normativa sobre a recuperação de custos e a viabilidade financeira evoluiu ao longo do tempo para se tornar cada vez mais empírica e pragmática.

Ao longo das duas últimas décadas, surgiram pesquisas com o intuito de debater e estudar os desafios e alternativas da supervisão financeira e do desempenho econômico-financeiro sob as perspectivas de vários setores econômicos regulados em diferentes países (Borio, 2003; Solver, 2005; Wymeersch, 2007; Treasury, 2009; Čihák \& Podpiera, 2009; Pan, 2010; Hilbers, Raaijmakers, Rijsbergen, \& De Vries, 2013; Hu, Yin, \& Zheng, 2016; Ringe, Morais, \& Ramos Munoz, 2019; Lowry \& Makos, 2020). De forma mais específica, Ergas e Small (2001), Pombo (2001), Arocena e Price (2002), Mehdi, Fetz e Filipini, (2007), Ribeiro et al. (2012), Braga et al. (2016), Huenteler et al. (2017) estudaram os modelos e sistemáticas adotados na supervisão financeira relacionados ao setor elétrico.

No Brasil, também destacam-se alguns estudos que analisaram a supervisão financeira por meio de indicadores de desempenho nos mercados regulados de seguros, seguros de saúde, instituições de ensino, distribuição de energia e instituições bancárias (Bezerra \& Corrar, 2006; Soares, 2006; Breitenbach, Alves, \& Diehl, 2010; Mendonça, Souza, \& Campos, 2016). A publicação de trabalhos relacionados à temática da supervisão econômico-financeira do setor de energia no Brasil também avançou na última década, sobretudo os trabalhos quantitativos (Bomfim, Almeida, Gouveia, Macedo, \& Marques, 2011; Brandão et al., 2016; Peris, 2016; Scalzer, Rodrigues, \& Macedo, 2015; Boente \& Lustosa, 2016; Scalzer, 2017, Jacob \& Bragança, 2017). Nessa literatura, predominam os estudos que utilizam os indicadores financeiros para verificar o desempenho de setores, organizações ou divisionais.

Este trabalho se diferencia dos demais, pois não foram verificados, até então, trabalhos com o objetivo de analisar a nova proposta sistemática de supervisão econômico-financeiro da ANEEL por meio do conjunto de indicadores propostos pelas notas técnicas. Ou seja, este parece ser o primeiro estudo que compara os indicadores financeiros de endividamento estudados na literatura com o indicador correspondente da ANEEL. Sendo assim, o objetivo principal deste trabalho é: verificar se o indicador de endividamento proposto pela Agência Nacional de Energia Elétrica (ANEEL) apresenta uma relação latente com os indicadores de endividamento utilizados pela literatura. Foram analisados dez indicadores de endividamento; nove mencionados pela literatura e um conforme a proposta da ANEEL. A base de dados foi extraída 
do site da agência. A relação entre os indicadores foi feita por meio da análise fatorial para agrupar variáveis proxy de endividamento e identificar se o indicador da ANEEL apresenta relação latente com os indicadores propostos pela literatura.

A relevância deste trabalho se baseia na necessidade tanto de estudar e desenvolver o modelo regulatório quanto de comparar a teoria debatida na literatura com a prática de avaliação do endividamento nas empresas no setor regulado de energia elétrica. Dessa forma, pode-se destacar contribuições tanto para a literatura como para a prática.

A contribuição deste trabalho para a literatura se destaca ao analisar se a estrutura dos indicadores já estudados em trabalhos anteriores manifesta relações latentes com o indicador apresentado pela ANEEL. Sob essa perspectiva, este estudo se insere no meio acadêmico como uma forma de identificar se esses indicadores utilizados pela literatura se alinham com o indicador de endividamento utilizado pela agência reguladora do setor de energia. Então, este trabalho se insere na linha de estudos empíricos que buscam avaliar se a literatura analisa as entidades de forma parecida com as agências que as avaliam, sua principal stakeholder.

Este trabalho também contribui para uma das questões mais complexas do SEB, as práticas de supervisão financeira mais propícias para que as operadoras de distribuição de energia tenham suas concessões renovadas (ANEEL, 2015). Ou seja, os estudos do endividamento e, consequentemente, da sustentabilidade financeira se mostram relevantes para a análise da situação financeira dessas empresas.

Por fim, este estudo se propõe a contribuir para a racionalidade acerca do indicador de endividamento proposto pela ANEEL. Espera-se colaborar com a ANEEL no desenvolvimento dos critérios de seleção e na aplicação de novos indicadores. Além disso, de forma mediata, este estudo espera ajudar operadores, reguladores e formuladores de políticas públicas nos constantes desafios relacionados ao aperfeiçoamento da modelagem e sistematização da supervisão econômico-financeira das operadoras. Como consequência, é possível que, no futuro, este estudo possa auxiliar na comparabilidade, confiabilidade, especificidade e regulação do indicador de endividamento (ANEEL, 2014).

\section{REFERENCIAL TEÓRICO}

\subsection{Análises de indicadores de desempenho e de modelos de supervisão econômico-financeira no setor elétrico}

Existem trabalhos na literatura que estudam os modelos e sistemáticas desenvolvidos ou adotados na supervisão financeira relacionada ao setor elétrico. Os trabalhos que se destacam são os de Ergas e Small (2001), Arocena e Price (2002), Mehdi et al. (2007), Jamasb e Pollitt (2008), Braga et al. (2016) e Huenteler et al. (2017). Dentre outras análises, esses estudos verificaram as escolhas entre os modelos de price-cap e a taxa de retorno, a forma de avaliação de desempenho, o benchmarking de eficiência ou as empresas de referência e, também, as perspectivas da Regulação Baseada em Desempenho (PBR). Um entendimento comum proporcionado por estes trabalhos é o de que a supervisão financeira tem origem na modelagem da arquitetura regulatória. Ou seja, o desempenho das entidades de energia elétrica está atrelado aos modelos de regulação, pois, de acordo com os autores, é assim que essas empresas guiam o negócio para se gerar desempenho. De forma geral, esses modelos ou sistemáticas de supervisão são compostos por diversas dimensões - dentre elas, a financeira - avaliadas por indicadores de desempenho.

Alguns estudos internacionais se destacam ao analisar os indicadores de desempenho das entidades de energia elétrica em diversos países, tais como Meenakumari e Kamaraj (2008), Tallapragada et al. (2009), e Kishore e Varun Kumar (2018). No Brasil, destacam-se alguns estudos acerca dos indicadores de desempenho das operadoras. Utilizando métodos quantitativos Bomfim et al. (2011), Brandão et al. (2016), Peris (2016), Scalzer et al. (2015), Boente e Lustosa 
(2016), Scalzer (2017) e Jacob e Bragança (2017) avaliaram o desempenho econômico-financeiro de operadoras do setor de energia.

Deve-se citar também que há contribuições acadêmicas que se dedicaram a sugerir modelos e/ou indicadores de desempenho na análise das entidades do setor elétrico, como as de Carregaro (2003), Campos (2005) e Ribeiro et al. (2012) e Caldeira (2013). É possível verificar ainda propostas metodológicas diferenciadas, tais como a de Kuosmanen et al. (2013), que analisaram a introdução do método StoNED para estimar o custo operacional eficiente das distribuidoras de energia elétrica por ocasião das reformas de supervisão do setor na Finlândia em 2012. Em outros países, para a mesma estimativa, os reguladores desse setor aplicam outros métodos, como a análise por envoltória de dados (DEA) ou análise de fronteira estocástica (SFA). Os autores compararam os impactos de escolhas metodológicas nas estimativas de eficiência de custo e custo aceitável. Embora as estimativas de eficiência sejam altamente correlacionadas, as metas de custo revelam grandes diferenças.

Todavia, nenhum dos trabalhos citados analisaram as possíveis interações entre a análise de desempenho tradicional, baseada na literatura, e os modelos ou sistemas regulatórios de supervisão de desempenho, sobretudo do novo modelo regulatório proposto pela ANEEL. Sendo assim, é possível destacar que os trabalhos encontrados na literatura se afastam da análise de desempenho utilizada pela ANEEL para avaliar as empresas de energia elétrica. Porém, é possível observar que a sistematização de indicadores de desempenho construída pela ANEEL, conforme a Nota Técnica 111/2016, segue a lógica do setor e, em todos os indicadores, há a inclusão de componentes específicos (setorizados) na fórmula de cálculo, apontando para um possível distanciamento dos resultados comparados aos da literatura. Neste trabalho os indicadores da literatura são comparados aos indicadores da ANEEL e a composição de cada um é apresentada sessão de metodologia.

\subsection{Análise fatorial em estudos sobre indicadores econômico-financeiros de entidades de setores regulados}

A análise fatorial é uma técnica estatística multivariada com uso crescente em todas as áreas de pesquisa relacionadas a negócios (Hair, Black, Babin, Anderson, \& Tatham, 2009). Segundo os autores, à medida que o número de variáveis a serem consideradas em técnicas multivariadas aumenta, também aumenta a necessidade de se obter maior conhecimento da estrutura e das inter-relações das variáveis. A análise fatorial foi utilizada como técnica em alguns trabalhos que estudaram os indicadores de desempenho de empresas em setores regulados, tais como os de Bezerra e Corrar (2006), Soares (2006), Bomfim, Macedo e Marques (2013) e Mendonça et al. (2016). Esses trabalhos utilizam a metodologia quantitativa de análise fatorial para verificar o agrupamento de indicadores de desempenho observados na literatura.

Bezerra e Corrar (2006) utilizaram a análise fatorial para identificar os principais indicadores na avaliação do desempenho financeiro nas empresas do setor regulado de seguros. Mais especificamente, o objetivo do trabalho foi propor uma metodologia que diminui o grau de subjetividade na escolha dos indicadores que deverão compor a avaliação das empresas e permitiu uma análise simultânea do comportamento de vários indicadores. Neste caso, a análise fatorial serviu como uma forma de criar critérios de seleção dos indicadores financeiros.

Soares (2006) também analisou indicadores de desempenho com o intuito de comparar os indicadores preconizados pela literatura e os indicadores utilizados na regulação das entidades no setor de saúde suplementar no Brasil. Os resultados obtidos com base nos indicadores adotados pela literatura e pela ANS mostraram que ambos são relevantes para avaliar e classificar o desempenho das operadoras de planos de saúde (OPS), pois os indicadores seguem um padrão de comportamento parecido, demonstrando que há relações latentes entre eles.

Bomfim et al. (2013) utilizaram a técnica de analise fatorial como uma técnica para apontar os indicadores financeiros e operacionais mais relevantes na avaliação de desempenho de empresas no setor petrolífero. Os autores destacaram assim que os indicadores estão relacionados, 
respectivamente, à lucratividade das transações comerciais, à alavancagem financeira e operacional, à continuidade das operações e à capacidade de geração futura de valor das organizações pesquisadas

Mendonça et al. (2016) utilizaram a análise fatorial para identificar os indicadores econômico-financeiros mais relevantes para avaliar o desempenho das 118 instituições do setor bancário com atividades no Brasil nos anos de 2011 a 2014. Os resultados mostram os oito indicadores mais relevantes para avaliar o desempenho dessas instituições e que esses também podem ser substituídos por três fatores que explicam cerca de $89,23 \%$ do intervalo geral de dados.

Por fim, pode-se destacar que este trabalho se insere na lacuna da literatura, que ainda não utilizou a técnica de análise fatorial para analisar os indicadores de desempenho no setor regulado de energia. Portanto, este estudo parece ser o primeiro a agrupar e comparar os indicadores preconizados pela literatura e os indicadores da agência reguladora por meio da análise fatorial, seguindo a linha de pesquisa dos artigos supracitados.

\section{PROCEDIMENTOS METODOLÓGICOS}

Esta pesquisa foi classificada de acordo com Richardson (1999) e Gil (1999). Quanto à natureza dos objetivos, a pesquisa é explicativa e descritiva, e não experimental quanto ao método. Quanto às técnicas de coletas de dados, classifica-se como bibliográfica para a construção do referencial teórico e documental para a coleta das variáveis de pesquisa no site da ANEEL, caracterizando-se assim, como uma pesquisa ex-post facto. Quanto à temporalidade, a pesquisa classifica-se como longitudinal, analisando um período de nove anos, de 2011 a 2018. Quanto à abordagem, este estudo é classificado com quantitativo, pois com base em Fávero e Belfiore (2017), utiliza a análise fatorial como técnica estatística multivariada de análise de dados. Por fim, com relação ao tipo de amostragem, caracteriza-se como sendo não probabilística intencional das empresas distribuidoras do setor de energia do Brasil.

Com base no objetivo deste trabalho, que consiste em verificar se o indicador de endividamento proposto pela Agência Nacional de Energia Elétrica (ANEEL) apresenta uma relação latente com os indicadores de endividamento utilizados pela literatura, utilizou-se o método de análise fatorial. A ideia deste trabalho é agrupar indicadores que apresentam relações entre si com o intuito de identificar como o indicador de endividamento utilizado pela ANEEL se comporta em uma análise conjunta com os demais indicadores de endividamento utilizados pela literatura. A amostra deste estudo não é balanceada, pois ao longo do tempo algumas empresas foram consolidadas. Ou seja, o número de empresas varia a cada ano, com a amostra total composta por 64 empresas distribuidoras do setor de energia elétrica no Brasil cujos dados financeiros foram obtidos por meio das demonstrações contábeis disponibilizados pela ANEEL entre o período de 2011 a 2018.

\subsection{Descrição das Variáveis}

Os indicadores de endividamento propostos pela literatura estão listados na Tabela 1 com a seguinte configuração: na primeira coluna, a descrição e o label da utilizado nas análises estatísticas; na segunda, a fórmula; e, na terceira, uma descrição do seu alcance de avaliação.

A ANEEL avalia o endividamento das distribuidoras por meio do indicador conforme a Nota Técnica 111/2016 (ANEEL, 2016a). Neste indicador, encontram-se variáveis com características particulares do setor regulado, como a Dívida Líquida Regulatória (DLR) e a Quota de Reintegração Regulatória (QRR). 


\section{Tabela 1}

\section{Indicadores de endividamento propostos pela literatura}

\begin{tabular}{|c|c|c|c|}
\hline & IndicadorlVariável & Fórmula & Alcance \\
\hline \multirow[t]{2}{*}{1 . } & \multirow{2}{*}{$\begin{array}{l}\text { Participação de } \\
\text { Capital de Terceiros } \\
\text { (PCT). }\end{array}$} & $\begin{array}{c}\text { (PassivoCirculante + } \\
\text { PassivoNãoCirc.) }\end{array}$ & \multirow{2}{*}{$\begin{array}{l}\text { Quanto maior, pior o desempenho. Indica a } \\
\text { dependência da empresa em relação aos recursos } \\
\text { externos. }\end{array}$} \\
\hline & & Passivo Total & \\
\hline \multirow[t]{2}{*}{2.} & Composição do & Passivo Circulante & \multirow{2}{*}{$\begin{array}{l}\text { Quanto menor, melhor o desempenho. Informa qual } \\
\text { o percentual de obrigações de curto prazo em } \\
\text { relação às obrigações totais, isto é, qual a parcela } \\
\text { das dívidas que vencem no curto prazo. }\end{array}$} \\
\hline & & $\begin{array}{c}\text { (PassivoCirculante+PassivoNã } \\
\text { oCirculante) }\end{array}$ & \\
\hline & $\begin{array}{l}\text { Grau de } \\
\text { endividamento (GE). }\end{array}$ & $\begin{array}{c}\text { (Passivo Circulante + Passivo } \\
\text { Não Circ.) }\end{array}$ & \multirow{2}{*}{$\begin{array}{l}\text { Quanto menor, melhor o desempenho. Indica } \\
\text { quanto a empresa tomou de capitais de terceiros } \\
\text { para cada } 100 \text { unidades monetárias de capital } \\
\text { próprio investido. }\end{array}$} \\
\hline & & (Patrimôn & \\
\hline & $\begin{array}{l}\text { Imobilização do } \\
\text { Patrimônio Líquido }\end{array}$ & Ativo & \multirow{2}{*}{$\begin{array}{l}\text { Quanto menor, melhor o desempenho. Mostra } \\
\text { quanto a empresa aplicou no ativo fixo } \\
\text { (imobilizado+ investimentos }+ \text { intangível) para } \\
\text { cada } 100 \text { unidades monetárias de patrimônio } \\
\text { líquido. }\end{array}$} \\
\hline & & Patri & \\
\hline & & Passivo & \multirow{2}{*}{$\begin{array}{l}\text { Quanto maior, pior o desempenho. Indica a as } \\
\text { obrigações da empresa no curto prazo em relação a } \\
\text { todas as obrigações da mesma. }\end{array}$} \\
\hline & $\begin{array}{l}\text { capitais d } \\
\text { no curto } 1 \\
(\mathrm{PTCP}) \text {. }\end{array}$ & $\begin{array}{r}\text { (Passivo Circula } \\
\text { Não Circulante } \\
\text { Líquic }\end{array}$ & \\
\hline & $\begin{array}{l}\text { Cobertura dos } \\
\text { encargos financeiros }\end{array}$ & $\begin{array}{r}\text { (Lucro Ope } \\
\text { Fin.+Out }\end{array}$ & \multirow{2}{*}{$\begin{array}{l}\text { Quanto maior, melhor o desempenho. Indica quanto } \\
\text { à empresa gera de receitas e lucro por unidade } \\
\text { monetária de despesas financeiras. }\end{array}$} \\
\hline & (CEF & Despesas & \\
\hline & Índice de cobertura & & \multirow{6}{*}{$\begin{array}{l}\text { Quanto mais elevado, maior o retorno } \\
\text { proporcionado por fontes onerosas. Quanto maior o } \\
\text { índice, maior a capacidade da empresa em honrar } \\
\text { com seus compromissos com credores. Avalia a } \\
\text { capacidade da empresa de gerar recursos para fazer } \\
\text { frente aos encargos de sua dívida. }\end{array}$} \\
\hline & de juros (EBT1). & (Despesas fina & \\
\hline & \multirow{2}{*}{$\begin{array}{l}\text { Índice de cobertura } \\
\text { de juros (EBT2). }\end{array}$} & EBI & \\
\hline & & Despesas financeiras & \\
\hline \multirow{2}{*}{\multicolumn{2}{|c|}{$\begin{array}{ll}\text { 9. } & \text { EFD/EBITDA } \\
& \text { (EBT3). }\end{array}$}} & $\begin{array}{c}\text { (Empréstimos e financiamentos } \\
\text {-Disponibilidades) } \\
\end{array}$ & \\
\hline & & & \\
\hline
\end{tabular}

Fonte: Variáveis criada com base em Ribeiro e Santos (2004), Lima e Freire (2008), Ribeiro et al. (2012), Delen et al. (2013), Ozório (2015) e Castro et al. (2017).

A DLR corresponde aos ativos e os passivos financeiros setoriais, que são os passivos onerosos formados por empréstimos, financiamentos e debêntures; o passivo atuarial (previdência privada e demais benefícios pós-emprego); o parcelamento de tributos; os instrumentos financeiros derivativos; os tributos em atraso; os custos setoriais renegociados; os encargos setoriais em atraso renegociados; os passivos financeiros setoriais; e o suprimento de energia elétrica para revenda curto prazo sem cobertura tarifária - subtraídos por caixa e equivalentes de caixa; investimentos temporários; instrumentos financeiros derivativos; ativos financeiros setoriais; e benefícios pósemprego (ANEEL, 2016b). Com relação à QRR, esta considera a depreciação e a amortização dos investimentos realizados, visando recompor os ativos relacionados à prestação do serviço ao longo da sua vida útil (ANEEL, 2009)

A Tabela 2 apresenta a descrição do indicador de endividamento proposto pela ANEEL com a seguinte configuração: na primeira coluna a descrição e o label da variável utilizada nas análises estatísticas; na segunda, a fórmula; e, na terceira, uma descrição do seu alcance de avaliação. 
Tabela 2

Indicadores de Endividamento Propostos pela ANEEL

\begin{tabular}{c|c|l}
\hline \multicolumn{1}{c|}{$\begin{array}{c}\text { IndicadorlVariável } \\
\begin{array}{l}\text { 10. Endividamento } \\
\text { (END). }\end{array}\end{array}$} & Fórmula & \multicolumn{1}{c}{ Alcance } \\
\cline { 2 - 3 } & EBITDA ajustado UDM-QRR UDM & $\begin{array}{l}\text { Quanto maior pior. É a medida relativa do } \\
\text { caixa livre disponível para pagamento das } \\
\text { dívidas subtraído do montante necessário a } \\
\text { manutenção dos ativos. UDM: últimos doze } \\
\text { meses. }\end{array}$ \\
\hline
\end{tabular}

Fonte: Nota Técnica n ${ }^{\circ} 111$ (ANEEL, 2016a).

\subsection{Estruturação do método de análise fatorial}

Inicialmente, foi operacionalizada uma análise descritiva dos indicadores (Tabela 3). Para realizar uma análise fatorial recomenda-se ter, no mínimo, cinco vezes mais observações do que o número de variáveis a serem analisadas, sendo que o ideal seria uma proporção de 10 para um (Hair, et al., 2009). Considerando que a amostra geral está composta por 480 observações, a proporção é de aproximadamente 37,8 .

A análise descritiva também aponta para a possibilidade da existência de outliers. Assim, foram analisadas todas as variáveis pelo método box plot (omitidos por questão de espaço) e verificou-se a presença de outliers em todas estas. Como este trabalho se propõe a verificar o setor de forma geral, optou-se por não operacionalizar um tratamento desses outliers, mantendo-se a originalidade da base.

O primeiro passo do processo de análise fatorial é a análise de correlação entre as variáveis estudadas (Tabela 4). Como a matriz de correlação apresentou variáveis relacionadas em alto grau, tanto positivamente como negativamente, e que atendem ao grau de significância de $5 \%$ entre variáveis da ANEEL e a literatura, foi possível realizar dois testes de análise fatorial.

O primeiro teste consistiu na aplicação da análise fatorial com todas as variáveis. No segundo teste, foram adicionadas somente as variáveis END e aquelas que se relacionaram significativamente com END como uma forma de verificar se as END se agrupariam com as variáveis que apresentaram correlação significante, mesmo em uma análise mais minuciosa.

Primeiro, aplicou-se, a análise fatorial com os dez indicadores (Tabela 5). Para formar um fator, o autovalor correspondente deve ser maior que 1, de acordo com o critério da Raiz Latente (critério de Kaiser). Com base nos resultados desta primeira análise, em vez de se trabalhar com os dez indicadores de endividamento, foram utilizados somente quatro fatores que englobam os dez indicadores, uma vez que são responsáveis pela explicação de $86,07 \%$ da associação total entre os dados. Assim, identifica-se um número mínimo de fatores que explicam a parcela máxima da variância dos indicadores. Esses fatores representam cinco dimensões subjacentes aos dados, úteis na análise de endividamento das distribuidoras, objeto deste estudo.

Foram analisadas as cargas fatoriais da análise fatorial. As cargas fatoriais são as correlações de Pearson entre cada variável e cada fator; o somatório dos quadrados dessa relação em cada linha da matriz, variável e fator, determina a comunalidade. Além disso, na matriz de cargas fatoriais, há a coluna uniqueness, ou exclusividade, cujos valores representam, para cada variável, o percentual de variância perdida para compor os fatores extraídos. A diferença entre 1 e esses valores resulta na comunalidade, que representa o percentual de variância compartilhada de cada variável original. Segundo Hair et al. (2009), uma diretriz estatística para a consideração prática é um nível mínimo de 0,5 para comunalidades; todas as variáveis analisadas superam este patamar. Esta análise pode identificar que não houve a necessidade de exclusão de variáveis para o aumento da explicação da variância. O teste de KMO aponta a adequação global da análise fatorial; recomenda-se um mínimo de 0,5 para que a análise seja considerada apropriada (Fávero \& Belfiore, 2017).

A fim de identificar os indicadores de endividamento mais significativos para a avaliação das distribuidoras e estabelecer a composição dos quatro fatores gerados pela análise fatorial, foi 
operacionalizada a análise da matriz de componentes rotacionados gerada pelo método Varimax. A matriz de componentes, após a rotação ortogonal, visa extremar ou maximizar os valores das cargas fatoriais de cada variável a um determinado fator, de modo que cada variável se associe a apenas um fator.

\section{RESULTADOS}

O SEB, particularmente o segmento de distribuição, apresenta uma heterogeneidade de características contratuais referentes à concessão e às operações financeiras. Há pequenas, médias e grandes operadoras, operadoras controladas pelo capital público e outras pelo capital privado, operações complexas de grandes extensões territoriais e operações tipicamente urbanas. Nesse contexto, é possível que a base de dados reflita estas características. Na análise descritiva dos indicadores (Tabela 3), verifica-se que o número de observações das variáveis (n) altera de tamanho, sendo 503 para o maior e 480 para o menor número de indicadores. Dessa forma, o pareamento dos dados, dada à ocorrência, limitou a amostra geral (n) utilizada para as análises em 480 observações.

Tabela 3

Análise descritiva dos indicadores

\begin{tabular}{lrrrrrrrr}
\hline \multicolumn{1}{c}{ Var. } & N.Obs & \multicolumn{1}{c}{ Média } & \multicolumn{1}{c}{ p50 } & \multicolumn{1}{c}{ Des. Pad. } & \multicolumn{1}{c}{ Mín. } & \multicolumn{1}{c}{ Máx. } & \multicolumn{1}{c}{ Amplitude } & \multicolumn{1}{c}{ Variância } \\
\hline PCT & 503 & 0,635 & 0,537 & 0,478 & 0,085 & 4,523 & 4,438 & 0,228 \\
CE & 503 & 0,519 & 0,477 & 0,183 & 0,13 & 1,000 & 0,861 & 0,033 \\
GE & 503 & 1,866 & 1,942 & 33,248 & $-678,21$ & 217,883 & 896,094 & 1105,459 \\
IPL & 503 & 1,933 & 2,176 & 24,883 & $-523,377$ & 118,751 & 642,128 & 619,146 \\
PTCP & 503 & 0,388 & 0,327 & 0,311 & 0,090 & 3,537 & 3,447 & 0,097 \\
CEF & 503 & $-2,607$ & $-1,941$ & 4,793 & $-66,174$ & 32,406 & 98,580 & 22,97 \\
EBT1 & 503 & 1,016 & 0,726 & 3,767 & $-44,612$ & 53,714 & 98,326 & 14,19 \\
EBT2 & 503 & 1,679 & 1,231 & 4,223 & $-42,821$ & 58,103 & 100,924 & 17,837 \\
EBT3 & 480 & 1,041 & 1,412 & 11,086 & $-169,266$ & 103,799 & 273,065 & 122,893 \\
END & 503 & 29,43 & 2,648 & 638,08 & $-1046,7$ & 14246,0 & 15292,7 & 407139,8 \\
\hline
\end{tabular}

Nota. PCT: Participação de Capital de Terceiros; CE: Composição de Endividamento; GE: Grau de Endividamento; IPL: Imobilização do Patrimônio Líquido; PTCP: Participação de capital de terceiros no curto prazo; CEF: Cobertura de encargos financeiros; EBT1: Ebit dividido pelas despesas financeiras líquidas; EBT2 Ebitda dividido pelas despesas financeiras líquidas; EBT3: EFD dividido pelo EBITDA; END: Endividamento.

Os resultados da análise descritiva, apresentados na Tabela 3, parecem corroborar com as diferentes características das operadoras de distribuição supracitadas, pois os valores de amplitude - diferença entre os valores mínimo e máximo - é relevante. Além disso, os desvios-padrões extrapolam a média, com a exceção apenas dos indicadores PCT, CE e PTCP, apontando alta discrepância entre os valores da amostra e demonstrando a variação de tamanho entre as empresas do setor de energia elétrica. Além disso, é importante ressaltar que, embora reconheçamos as limitações dos resultados deste trabalho perante a análise das observações com PL negativo, optouse por manter as observações da forma como a ANEEL trata em sua base de dados, ou seja, considerando toda a amostra. A Tabela 4 apresenta a análise de correlação das variáveis estudadas. 
Tabela 4

Matriz de Correlação com doze variáveis

\begin{tabular}{|c|c|c|c|c|c|c|c|c|c|c|}
\hline Ind. & PCT & CE & GE & IPL & PTCP & CEF & EBT1 & EBT2 & EBT3 & END \\
\hline PCT & 1,000 & & & & & & & & & \\
\hline $\mathrm{CE}$ & $-0,117 *$ & 1,000 & & & & & & & & \\
\hline GE & $-0,866^{*}$ & $0,193^{*}$ & 1,000 & & & & & & & \\
\hline IPL & $-0,123^{*}$ & $0,123 *$ & $0,110 *$ & 1,000 & & & & & & \\
\hline PTCP & $0,209 *$ & $-0,369 *$ & $-0,427^{*}$ & $-0,203^{*}$ & 1,000 & & & & & \\
\hline $\mathrm{CEF}$ & $0,117 *$ & $-0,031$ & $0,132 *$ & $-0,034$ & $0,249 *$ & 1,000 & & & & \\
\hline EBT1 & $-0,067$ & 0,053 & $0,113^{*}$ & 0,007 & $0,343^{*}$ & $0,864^{*}$ & 1,0000 & & & \\
\hline EBT2 & $-0,045$ & 0,040 & 0,081 & -0.048 & $0,328 *$ & $0,876^{*}$ & $0,939 *$ & 1,000 & & \\
\hline EBT3 & $-0,397 *$ & 0,013 & $0,217^{*}$ & $0,237 *$ & 0,038 & 0,045 & 0,023 & 0,015 & 1,000 & \\
\hline END & $-0,405^{*}$ & $0,232 *$ & $-0,538 *$ & 0,083 & 0,004 & 0,674 & $-0,062$ & $-0,605$ & $0,720 *$ & 1,000 \\
\hline
\end{tabular}

Nota. * para 5\%. PCT: Participação de Capital de Terceiros; CE: Composição de Endividamento; GE: Grau de Endividamento; IPL: Imobilização do Patrimônio Líquido; PTCP: Participação de capital de terceiros no curto prazo; CEF: Cobertura de encargos financeiros; EBT1: Ebit dividido pelas despesas financeiras líquidas; EBT2 Ebitda dividido pelas despesas financeiras líquidas; EBT3: EFD dividido pelo EBITDA; END: Endividamento.

Verifica-se na Tabela 4 que as variáveis PCT, CE, GE e EBT3 apresentam correlação significativa com a variável END. Sendo assim, a matriz apresenta o primeiro resultado relevante para este trabalho, pois há correlações estatisticamente significantes de $5 \%$, positivas ou negativas, entre o indicador proposto pela ANEEL (END) e os demais indicadores verificados na literatura.

Os resultados da primeira análise fatorial com dez indicadores se encontram na Tabela 5, que apresenta os autovalores da matriz de correlações com os respectivos percentuais de variância compartilhada das variáveis originais dos fatores não rotacionados.

Tabela 5

Total da Variância Explicada

\begin{tabular}{lrrrrrr}
\hline \multicolumn{1}{c}{ Fator } & \multicolumn{3}{c}{ Dados originais } & \multicolumn{3}{c}{ Fatores rotacionados } \\
& Autovalores & \%variância & $\begin{array}{c}\text { \%variância } \\
\text { acumulada }\end{array}$ & Variância & $\begin{array}{c}\text { \% de } \\
\text { variância }\end{array}$ & $\begin{array}{c}\text { \% } \\
\text { acumulada }\end{array}$ \\
\hline Fator1 & 2,99259 & 0,2993 & 0,2993 & 2,83705 & 0,2837 & 0,2837 \\
Fator2 & 2,61940 & 0,2619 & 0,5612 & 2,63182 & 0,2632 & 0,5469 \\
Fator3 & 1,85948 & 0,1859 & 0,7471 & 1,87493 & 0,1875 & 0,7344 \\
Fator4 & 1,13595 & 0,1136 & 0,8607 & 1,26361 & 0,1264 & 0,8607 \\
Fator5 & 0,77660 & 0,0777 & 0,9384 & & & \\
Fator6 & 0,29314 & 0,0293 & 0,9677 & & & \\
Fator7 & 0,13196 & 0,0132 & 0,9809 & & & \\
Fator8 & 0,10477 & 0,0105 & 0,9914 & & & \\
Fator9 & 0,04787 & 0,0048 & 0,9914 & & & \\
Fator10 & 0,03824 & 0,0038 & 1,0000 & & & \\
\hline
\end{tabular}

Nota. Dados da Pesquisa. PCT: Participação de Capital de Terceiros; CE: Composição de Endividamento; GE: Grau de Endividamento; IPL: Imobilização do Patrimônio Líquido; PTCP: Participação de capital de terceiros no curto prazo; CEF: Cobertura de encargos financeiros; EBT1: Ebit dividido pelas despesas financeiras líquidas; EBT2 Ebitda dividido pelas despesas financeiras líquidas; EBT3: EFD dividido pelo EBITDA; END: Endividamento.

Observa-se na Tabela 5 que o número de fatores formados e retidos foi igual a quatro. É possível verificar na coluna "\% acumulada", que o grau de explicação atingido por tais fatores é alto $(86,07 \%)$. Ou seja, os quatro fatores retidos representam 86,07\% da variância das dez variáveis.

Assim, identifica-se um número mínimo de fatores que explicam a parcela máxima da variância dos indicadores. Esses fatores representam cinco dimensões subjacentes aos dados, úteis na análise de endividamento das distribuidoras que são objeto deste estudo.

Na Tabela 6, observam-se as cargas fatoriais de cada variável. Os resultados indicaram que não houve a necessidade de exclusão de variáveis para o aumento da explicação da variância. 
Tabela 6

\section{Cargas Fatoriais e Comunalidades}

\begin{tabular}{lcccccc}
\hline Variável & Factor1 & Factor2 & Factor3 & Factor4 & Uniqueness & Comunalidade \\
\hline PCT & $-0,0593$ & $\mathbf{0 , 9 3 3 1}$ & $-0,1429$ & 0,0741 & 0,0999 & 0,9001 \\
CE & $-0,0481$ & 0,1436 & $\mathbf{0 , 8 5 7 8}$ & $-0,0351$ & 0,2399 & 0,7601 \\
GE & 0,0404 & $\mathbf{- 0 , 9 6 0 5}$ & 0,0657 & $-0,0122$ & 0,0713 & 0,9287 \\
IPL & 0,064 & 0,1077 & 0,1999 & $\mathbf{0 , 8 8 1 1}$ & 0,1679 & 0,8321 \\
PTCP & 0,0155 & 0,365 & $\mathbf{- 0 , 8 6 0 4}$ & $-0,2008$ & 0,086 & 0,914 \\
CEF & $\mathbf{0 , 9 6 0 7}$ & 0,0172 & $-0,0378$ & 0,0199 & 0,075 & 0,925 \\
EBT1 & $\mathbf{0 , 9 7 2 9}$ & $-0,0576$ & $-0,0195$ & 0,0353 & 0,0485 & 0,9515 \\
EBT2 & $\mathbf{0 , 9 7 7 3}$ & $-0,0455$ & 0,0075 & 0,0078 & 0,0428 & 0,9572 \\
EBT3 & -0.0029 & $\mathbf{- 0 , 5 5 6 4}$ & $-0,4408$ & 0,554 & 0,1893 & 0,8107 \\
END & 0.0272 & $\mathbf{0 , 5 9 8 2}$ & 0,3715 & $-0,3625$ & 0,3721 & 0,6279 \\
\hline
\end{tabular}

Nota. PCT: Participação de Capital de Terceiros; CE: Composição de Endividamento; GE: Grau de Endividamento; EBT3: EFD dividido pelo EBITDA; END: Endividamento.

O resultado da análise KMO foi de 0,623, apontando para a adequação global da análise fatorial. A partir da estatística de qui-quadrado do teste de esfericidade de Barlett, é possível afirmar, para o nível de significância de $1 \%$ e 45 graus de liberdade, que a matriz de correlações de Pearson é estatisticamente diferente da matriz identidade de mesma dimensão, visto que $\mathrm{x}^{2}$ apresentou valor de 1396,32 e p-valor de 0,000, ou seja, p-valor < 0,01. Esses testes apontam a adequação da análise fatorial para a análise e o tratamento dos dados.

Os resultados da análise da matriz de componentes rotacionados também podem ser verificados na Tabela 6 (valores em negrito). Em uma análise conjunta dos resultados apresentados nas tabelas 5 e 6, verifica-se que o Fator 1, composto pelos indicadores CEF, EBT1 e EBT2, é responsável por 28,37\% da variância dos indicadores (\% de variância, Tabela 5). Verifica-se também que o Fator 2, composto pelos indicadores PCT, GE, EBT3 e END, é responsável por 26,32\% da variância dos indicadores. Já o Fator 3, composto pelos indicadores CE e PTCP, é responsável por $18,75 \%$ da variância dos indicadores. Por fim, verifica-se que o Fator 4, composto pelo indicador IPL, explica sozinho $12,64 \%$ da variância dos indicadores.

Aplicou-se, então, a análise fatorial para os cinco indicadores, sendo eles: END e as quatro variáveis que se correlacionaram significativamente com ele: PCT, CE, GE e EBT3. Os resultados da Matriz de Variância Total Explicada estão na Tabela 7, a qual apresenta os autovalores da matriz de correlações com os respectivos percentuais de variância compartilhada das variáveis originais dos fatores não rotacionados.

Tabela 7

\section{Total da Variância Explicada}

\begin{tabular}{|c|c|c|c|c|c|c|}
\hline \multirow[b]{2}{*}{ Fator } & \multicolumn{3}{|c|}{ Dados originais } & \multicolumn{3}{|c|}{ Fatores rotacionados } \\
\hline & Autovalores & $\begin{array}{c}\% \\
\text { variância }\end{array}$ & $\begin{array}{l}\text { \% variância } \\
\text { acumulada }\end{array}$ & Variância & $\begin{array}{c}\text { \% de } \\
\text { variância }\end{array}$ & $\begin{array}{c}\% \\
\text { acumulada }\end{array}$ \\
\hline Fator1 & 2,92919 & 0,5858 & 0,5858 & 2,92919 & 0,5858 & 0,5858 \\
\hline Fator2 & 0,98920 & 0,1978 & 0,7837 & & & \\
\hline Fator3 & 0,79227 & 0,1585 & 0,9421 & & & \\
\hline Fator4 & 0,23272 & 0,0465 & 0,9887 & & & \\
\hline Fator5 & 0,05661 & 0,0113 & 1,0000 & & & \\
\hline
\end{tabular}

Nota. Dados da Pesquisa.

Observa-se na Tabela 7 que o número de fatores formados e retidos foi igual a um, atendendo assim ao critério da Raiz Latente. É possível verificar na coluna "\% acumulada" que o grau de explicação atingido por tais fatores é de mais da metade; assim, o fator retido representa $58,58 \%$ da variância das cinco variáveis.

Com base nos primeiros resultados, em vez de se trabalhar com os seis indicadores de endividamento, foi formado somente um fator que engloba os cinco indicadores, uma vez que são 
responsáveis pela explicação de 58,58\% da associação total entre os dados. Assim, identifica-se um número mínimo de fatores que explicam a parcela máxima da variância dos indicadores. Esses fatores representam uma das dimensões subjacentes aos dados, úteis na análise de endividamento das distribuidoras que são objeto deste estudo.

$\mathrm{Na}$ Tabela 8, observam-se as cargas fatoriais de cada variável. Como já abordado anteriormente, segundo Hair et al. (2009), uma diretriz estatística para a consideração prática dos valores é de um nível mínimo de 0,5 para comunalidades. Verifica-se na Tabela 8 , que todas as variáveis analisadas superam o valor de 0,5 , com exceção da variável $\mathrm{CE}$, a qual apresentou o valor de 0,281. Portanto, esta variável parece ser a que mais se afasta do comportamento apresentado pelas demais variáveis agrupadas no fator. Além de apresentar comunalidade menor que 0,5, a variável CE foi agrupada em um fator diferente na primeira análise fatorial apresentada neste artigo.

Tabela 8

\section{Cargas Fatoriais e Comunalidades}

\begin{tabular}{lccc}
\hline Variável & Factor1 & Uniqueness & Comunalidade \\
\hline PCT & 0,8144 & 0,3367 & 0,6633 \\
CE & 0,5301 & 0,7190 & 0,2810 \\
GE & $-0,8773$ & 0,2304 & 0,7696 \\
EBT3 & $-0,7954$ & 0,3674 & 0,6326 \\
END & 0,7633 & 0,4173 & 0,5827 \\
\hline
\end{tabular}

Nota. PCT: Participação de Capital de Terceiros; CE: Composição de Endividamento; GE: Grau de Endividamento; EBT3: EFD dividido pelo EBITDA; END: Endividamento.

O resultado da análise KMO foi de 0,6314, apontando que há adequação global da análise fatorial. A partir da estatística de qui-quadrado do teste de esfericidade de Barlett, é possível afirmar, para o nível de significância de $1 \%$ e 10 graus de liberdade, que a matriz de correlações de Pearson é estatisticamente diferente da matriz identidade de mesma dimensão, visto que $\mathrm{x}^{2}$ apresentou valor de 767,90 e p-valor de 0,000, ou seja, p-valor <0,01. Assim, esses testes apontam a adequação da análise fatorial para a análise e o tratamento dos dados. Como todas as variáveis foram agrupadas em um fator único, não foi feito o teste da análise fatorial com o fator rotacionado, pois os resultados seriam os mesmos.

Em uma análise conjunta dos resultados apresentados nas tabelas 5, 6, 7 e 8, verifica-se que o indicador da ANEEL, END, se agrupa com as variáveis utilizadas na literatura - PCT, GE e EBT. Esse agrupamento pode ser verificado em ambas as análises fatoriais, pois o END foi agrupado tanto na primeira análise com todas as variáveis incluídas no teste e quanto somente com aquelas que apresentaram correlação significante com END. Apesar de END apresentar correlação significante com a variável CE, a mesma não se agrupa à variável END na primeira análise e não apresenta valor de comunalidade alto no segundo teste da análise fatorial. Portanto, decidiu-se não identificar esta variável como uma variável que apresenta relação latente com END.

Com base nos resultados, é possível apontar que o indicador proposto pela ANEEL foi agrupado com outros indicadores de endividamento utilizados na pesquisa em contabilidade no setor de energia elétrica: PCT, GE e EBT3. Estes resultados respondem ao principal objetivo deste trabalho, que buscou avaliar a possibilidade do indicador de endividamento proposto pelo modelo regulador poder apresentar correlação ou uma relação latente com os identificados na literatura. Foram verificadas correlações significativas com END e foram encontradas relações latentes entre a variável de endividamento utilizada pela ANEEL e outros três indicadores preconizados pela literatura. Optou-se por não nomear os Fatores, já que o objetivo do trabalho não foi criar fatores com o intuito de substituir a análise dos indicadores, mas sim analisar a maneira com que os indicadores de endividamento utilizados na literatura se comportavam com relação à variável utilizada pela ANEEL. 
Este estudo compara os indicadores financeiros de endividamento estudados na literatura com o indicador de endividamento da ANEEL. A falta de estudos equivalentes não permitiu que os resultados sejam comparados com os de outros autores, sendo esta uma limitação da presente pesquisa.

\section{CONCLUSÕES}

O objetivo principal deste trabalho consistiu em verificar se o indicador de endividamento apurado nas demonstrações financeiras das distribuidoras e proposto pela nova sistemática de controle ANEEL pode estar correlacionado ou apresentar relação latente aos indicadores de endividamento utilizados pela literatura. Este trabalho verificou as possíveis relações entre os indicadores propostos pelo órgão regulador e os indicadores verificados na literatura.

De forma geral, os estudos que avaliam a relevância de indicadores de desempenho apontam que aqueles que são relacionados ao endividamento formam um dos grupo dos mais relevantes para a avaliação de desempenho das empresas nos segmentos regulados no Brasil (Bomfim et al., 2011; Ribeiro et al. 2012; Bomfim et al., 2013; Scalzer et al., 2015; Mendonça, Souza \& Campos, 2016; Mendonça et al, 2016; Scalzer, 2017). Essa importância também é dada pela ANEEL para os indicadores de endividamento.

A temática analisada por este trabalho enquadrou-se, na linha de pesquisa que inclui a análise da supervisão econômico-financeira. Essa linha abarca a modelagem e a sistemática da supervisão de setores reguladores e, por sua vez, também inclui os indicadores propostos pelas agências para acompanhar essas modelagens ou sistemáticas e auferir o desempenho das entidades.

Foi utilizada a análise fatorial, e a divisão dos indicadores entre os fatores apontou que há relações latentes entre o indicador de endividamento proposto pela ANEEL e três outros indicadores utilizados pela literatura. Esses resultados, em que é possível identificar essas relações latentes entre indicadores utilizados pela agência e pela literatura, também foram encontrados por Soares (2006) no setor de saúde. Sendo assim, é possível indicar que a literatura não se distancia da prática no quesito de analisar indicadores de desempenho em alguns setores regulados.

Pode-se concluir que, apesar de a ANEEL desenvolver o indicador de endividamento com o intuito de proceder uma análise setorial, este apresenta padrão parecido com outros indicadores utilizados pela literatura. Além de cumprir, principalmente, com a missão de gerar subsídios para o momento de renovação das concessões, o indicador de endividamento também apresenta relações latentes com alguns outros utilizados pela literatura. Assim, a inserção de componentes do SEB na composição do indicador proposto de endividamento (END), por exemplo, o QRR e a DLR, pode ser um diferencial na comparação com outros indicadores de endividamento, porém não parece ser fator fundamental para descolar o mesmo dos demais indicadores, tornando-o tipicamente setorial.

Dado os achados deste trabalho, seria pouco prudente apontar que o indicador de endividamento proposto pela ANEEL é relevante para a supervisão econômico-financeira das distribuidoras apenas por, tão somente, apresentar comportamento assemelhado ao encontrado na literatura. Porém, é possível apontar que, provavelmente, os indicadores que se agruparam com o indicador da ANEEL sejam os mais adequados para analisar o endividamento das empresas do setor de energia elétrica.

Na prática, os resultados deste trabalho podem contribuir para a racionalidade dos agentes envolvidos no setor de energia elétrica. Os stakeholders podem compreender melhor como os indicadores de endividamento comumente utilizados podem se relacionar com o indicador de endividamento utilizado pela agência reguladora, a ANEEL. Na literatura, este trabalho preenche a lacuna de comparar como os indicadores de endividamento utilizados pela literatura e pela agência reguladora no setor de energia elétrica. Contribui, assim, para o desenvolvimento de trabalhos que acompanham a dinâmica da supervisão econômico-financeira do SEB sob uma 
perspectiva empírico-quantitativa, analisando as interações das operadoras com a modelagem dos indicadores de desempenho tanto da literatura quanto da prática (agência reguladora).

Deve-se considerar que este trabalho possui limitações; dentre elas, existe a questão contratual do setor, que pode influenciar a análise de endividamento das entidades. O segmento de distribuição de energia elétrica possui inúmeras especificidades contratuais que não foram consideradas neste trabalho, tais como as operacionais, as financeiras, as acionárias (controle público ou privado) e os objetivos regulatórios, diferenciados para cada distribuidora. Há também problemas de qualidade da informação; verificam-se nas demonstrações financeiras, por exemplo, operadoras com patrimônio líquido negativo. Essas e outras eventuais especificidades não foram tratadas neste trabalho. Considera-se também, como limitação, a abordagem sob uma única perspectiva quantitativa, pois pode-se utilizar, por exemplo, a Análise Envoltória de Dados.

Como sugestões para pesquisas futuras, sugere-se que este trabalho seja aplicado observando-se algumas das especificidades do segmento de distribuição, como exemplo, operadoras segmentas pelo tamanho ou pela divisão entre controladas pelo capital público ou iniciativa privada. As análises também podem ser desenvolvidas sob uma nova perspectiva de metodologia quantitativa. Além disso, este trabalho analisou somente a dimensão do endividamento, sendo conhecido que a nova supervisão financeira das distribuidoras inclui outras dimensões. Sendo assim, é possível também analisar as outras dimensões, tais como a de investimentos e rentabilidade, cada uma com seus respectivos indicadores propostos pela ANEEL. Por fim, é possível apontar que a análise desenvolvida por este trabalho também pode ser feita em outros setores, utilizando os indicadores setoriais de outras agências reguladoras.

\section{REFERÊNCIAS}

ANEEL. (2009). Resolução Normativa $n^{o}$ 386/2009. Estabelece os conceitos gerais, as metodologias aplicáveis e os procedimentos para realização do Segundo Ciclo de Revisões Tarifárias Periódicas das concessionárias de serviço público de transmissão de energia elétrica. Brasília. 2009. Recuperado em 01 abril, 2019, de http://www2.aneel.gov.br/cedoc/ren2009386.pdf

ANEEL. (2014). Nota Técnica no 353/2014-SFF/ANEEL. Brasília. Recuperado em 29 março, 2019 http://www2.aneel.gov.br/aplicacoes/consulta_publica/documentos/NT_Indicadores\%20de\%2 0Sustentabilidade.pdf

ANEEL. (2015). Manual de Contabilidade do Setor Elétrico. Brasília.

ANEEL. (2016a). Nota Técnica no 67/2016-SFF/ANEEL - Instituição de indicadores públicos de sustentabilidade econômico-financeira. Brasília.

ANEEL. (2016b). Nota Técnica no 111/2016-SFF/ANEEL - Instituição de indicadores públicos de sustentabilidade econômico-financeira. Brasília.

Arocena, P., \& Price, C. W. (2002). Generating efficiency: economic and environmental regulation of public and private electricity generators in Spain. International Journal of Industrial Organization, 20(1), 41-69. doi: 10.1016/S0167-7187(00)00073-4

Bakovic, T., Tenenbaum, B., \& Woolf, F. (2002). Regulation by contract: a new way to privatize electricity distribution? The World Bank. 
Bezerra, F. A., \& Corrar, L. J. (2006). Utilização da análise fatorial na identificação dos principais indicadores para avaliação do desempenho financeiro: uma aplicação nas empresas de seguros. Revista Contabilidade \& Finanças, 17(42), 50-62. doi: 10.1590/S151970772006000300005

Boente, D. R., \& Lustosa, P. R. B. (2016). Um estudo comparativo da eficiência de distribuidoras brasileiras de energia elétrica privadas e públicas. Revista Ambiente Contábil - Universidade Federal do Rio Grande do Norte, 8(2), 263-286.

Bomfim, P. R. C. M., Almeida, R. S., Gouveia, V. A. L., Macedo, M. A. da S., \& Marques, J. A. V. C. (2011). Utilização de Análise Multivariada na Avaliação do Desempenho EconômicoFinanceiro de Curto Prazo: uma Aplicação no Setor de Distribuição de Energia Elétrica. Revista do Mestrado em Administração e Desenvolvimento Empresarial da Universidade Estácio de Sá, 15(1), 75-92.

Bomfim, P. R. C. M., Macedo, M. A. da S., \& Marques, J. A. V. da C. (2013). Indicadores Financeiros e Operacionais para a Avaliação de Desempenho de Empresas do Setor de Petróleo e Gás. Revista Ambiente Contábil, 16(1), 112-131.

Borio, C. (2003). Towards a macroprudential framework for financial supervision and regulation? CESifo Economic Studies, 49(2), 181-215.

Braga, K., Rufin, C., Brandão, R., \& Torres, E. (2016). Financial Regulation of the Electricity Distributors: Necessity and Feasibility. Energy Procedia, 106, 166-174. doi: 10.1016/j.egypro.2016.12.113

Brandão, R., Macedo, M. A., Ozorio, L. M., Scalzer, R., Tavares, A., Gomes, R. O., \& Pereira, R. M. R. Análise de Sustentabilidade Econômica e Financeira das Distribuidoras de Energia Elétrica no Brasil no período de 2009-2015. Congresso de Inovação Tecnológica em Energia Elétrica, 9.

Breitenbach, M., Alves, T. W., \& Diehl, C. A.(2010). Indicadores financeiros aplicados à gestão de instituições de ensino de educação básica. Contabilidade Vista \& Revista, 21(3).

Caldeira, T. C. M. (2013) Indicador de desempenho global das distribuidoras de energia elétrica. (Dissertação de mestrado) Mestrado profissional em Regulação e Gestão de Negócios, Universidade de Brasília, Brasília, DF. Brasil.

Campos, J. J. F. D. (2005). Sustentabilidade energética no brasil: proposta de indicadores para elaboração de relatórios de sustentabilidade por empresas do setor elétrico (Dissertação de mestrado). Fundação Getúlio Vargas, Escola de Administração de Empresas de São Paulo, São Paulo, SP, Brasil.

Carregaro, J. C. (2003). Proposta de indicadores de desempenho às distribuidoras de energia federalizadas do setor elétrico brasileiro. (Dissertação de mestrado) - Universidade Federal de Santa Catarina, Centro Tecnológico, Programa de Pós-Graduação em Engenharia de Produção, Florianópolis, SC, Brasil.

Castro, N., Brandão, R., Ozorio, L. M., Macedo, M. Á., Rodrigues, A., Torres Filho, E., Rufín C., Gomes, R. O., Scalzer, R., Tavares, A., Martins, N., Braga, K., \& Martins, N. (2017). 
Indicadores de Sustentabilidade Econômico-Financeira das Empresas de Distribuição de Energia Elétrica. Grupo de Estudos do Setor Elétrico UFRJ (GESEL). Rio de Janeiro.

Čihák, M., \& Podpiera, R. (2008). Integrated financial supervision: Which model? The North American Journal of Economics and Finance, 19(2), 135-152. doi: 10.1016/j.najef.2008.03.003

Delen, D., Kuzey, C., \& Uyar, A. (2013) Measuring firm performance using financial ratios: A decision tree approach. Expert Systems with Applications, 40(10), 3970-3983. doi: 10.1016/j.eswa.2013.01.012

Ergas, H., \& Small, J. (2001). Price caps and rate of return regulation. Network Economics Consulting Group.

Fávero, L. P. \& Belfiore, P. (2017). Manual de Análise de Dados. Rio de Janeiro: Elsevier.

Gil, A. C. (1999). Métodos e técnicas de pesquisa social. São Paulo: Atlas.

Hair, J. F., Black, B., Babin, B., Anderson, R. E., \& Tatham, R. L. (2009). Análise Multivariada de Dados. Porto Alegre: Bookman.

Hilbers, P., Raaijmakers, K., Rijsbergen, D. R., \& De Vries, F. (2013). Measuring the effects of financial sector supervision. DNB Working Papers 388, Netherlands Central Bank, Research Department.

Huenteler, J., Dobozi, I., Balabanyan, A., \& Banerjee, S. G. (2017). Cost recovery and financial viability of the power sector in developing countries: A literature review. doi: 10.1596/18139450-8287

Hu, B., Yin, Z., \& Zheng, L. (Eds.). (2016). Development of China's Financial Supervision and Regulation. Springer. doi: 10.1057 / 978-1-137-52225-2

Jacob, F. E., \& Bragança, G. G. F. D. (2017). Eficiência Financeira das Distribuidoras de Energia no Período de 2011 a 2014: uma análise comparativa usando DEA. Texto para discussão / Instituto de Pesquisa Econômica Aplicada. Brasília: Rio de Janeiro, RJ.

Jamasb, T., \& Pollitt, M. (2008). Security of supply and regulation of energy networks. Energy Policy, 36(12), 4584-4589. doi: 10.1016/j.enpol.2008.09.007

Kishore, L., \& Varun K., S. G. (2018). Operational and financial performance optimization of power distribution utilities in India. International Journal of Pure and Applied Mathematics, 119(15 Special Issue C), 1559-1566.

Kuosmanen, T., Saastamoinen, A., \& Sipiläinen, T. (2013). What is the best practice for benchmark regulation of electricity distribution? Comparison of DEA, SFA and StoNED methods. Energy Policy, 61, 740-750. doi: 10.1016/j.enpol.2013.05.091

Lima, L. C. G. B., \& Freire, F. S. (2008). Composição de Capital versus Desempenho Financeiro de Empresas de Distribuição de Energia Elétrica. Anais do Congresso Brasileiro de Custos$A B C$. 
Lowry, M. N., \& Makos, M. (2020). Performance-Based Regulation: Basic Features and Possible Applications to BC Hydro. Economist.

Meenakumari, R., \& Kamaraj, N. (2008). Measurement of relative efficiency of state-owned electric utilities in India using data envelopment analysis. Modern Applied Science, 2(5), 6171. DOI: $10.5539 /$ mas.v2n5p61

Mehdi, F., Fetz, A., \& Filippini, M. (2007). Benchmarking and regulation in the electricity distribution sector. In Servizi pubblici: nuove tendenze nella regolamentazione nella produzione e nel finanziamento, 54, 159-176. CEPE Working Paper.

Mendonça, D. J., Souza, J. A., \& Campos, R. S. (2016). Análise do desempenho dos maiores bancos brasileiros: um estudo com a análise envoltória de dados (DEA) aplicada a um conjunto de indicadores econômico-financeiros. Revista Observatório de La Economia Latinoamericana, Brasil, setembro.

Mendonça, D. J., Souza, J. A. E., Carvalho, F. D. M., \& Benedicto, G. C. (2016). Utilização da análise fatorial para identificação dos principais indicadores de avaliação de desempenho econômico-financeiro: uma aplicação em instituições financeiras bancárias. Congresso USP de Controladoria e Contabilidade, 16.

Ozório, L. D. M. (2015). Análise do Desempenho Econômico-Financeiro de Distribuidoras de Energia Elétrica no Brasil. Grupo de Estudos do Setor Elétrico UFRJ (GESEL).

Pan, E. J. (2010). Challenge of international cooperation and institutional design in financial supervision: beyond transgovernmental networks. Chi. J. Int'l L., 11, 243.

Peris, R. W. (2017). Análise do desempenho do setor elétrico no Brasil - 2010 a 2015. (Dissertação de Mestrado) Faculdade de Economia, Administração e Contabilidade, Universidade de São Paulo, São Paulo. doi: 10.11606/D.12.2017.tde-24032017-124815

Pombo, C. (2001). Regulatory reform in Colombia's electric utilities. The Quarterly Review of Economics and Finance, 41(5), 638-671. doi: 10.1016/S1062-9769(01)00098-9

Ribeiro, M. G. C., Macedo, M. Á. S., \& Marques, J. A. V. C. (2012). Análise da relevância de indicadores financeiros e não financeiros na avaliação de desempenho organizacional: um estudo exploratório no setor brasileiro de distribuição de energia elétrica. Revista de Contabilidade e Organizações, 6(15), 60-79.

Ribeiro, M. S., \& Santos, A. (2004). A remuneração dos capitais utilizados para financiamento dos ativos de empresas distribuidoras de energia elétrica medida por meio da DVA. $B B R$ Brazilian Business Review, 1(1), 17-30.

Richardson, R. J. (1999). Pesquisa social: métodos e técnicas. São Paulo: Atlas.

Ringe, W. G., Morais, L. S., \& Ramos Muñoz, D. (2019). A Holistic Approach to the Institutional Architecture of Financial Supervision and Regulation in the EU. Oxford Legal Studies Research Paper No. 56/2019, European Banking Institute Working Paper Series 2019 - n. 50. doi: dx.doi.org/10.2139/ssrn.3466649 
Scalzer, R. S. (2017). Insolvência Empresarial: Um Estudo sobre as Distribuidoras de Energia Elétrica Brasileiras. Universidade Federal do Rio de Janeiro, Rio de Janeiro.

Scalzer, R. S., Rodrigues, A., \& da Silva Macedo, M. Á. (2015). Insolvência empresarial: um estudo sobre as distribuidoras de energia elétrica brasileiras. Revista Contemporânea de Contabilidade, 12(27), 27-60.

Soares, M. A. (2006). Análise de indicadores para avaliação de desempenho econômicofinanceiro de operadoras de planos de saúde brasileiras: uma aplicação da análise fatorial. (Dissertação de Mestrado) Faculdade de Economia, Administração e Contabilidade, Universidade de São Paulo, São Paulo. doi: 10.11606/D.12.2006.tde-15122006-121519

Solver, T. (2005). Reliability in performance-based regulation. (Trabalho de conclusão de curso) Department of Electrical Systems, Royal Institute of Technology, Sweden. Recuperado em 01 maio, 2020, de https://www.diva-portal.org/smash/get/diva2:14598/FULLTEXT01.pdf

Tallapragada, V. S. N., Shkaratan, M., Izaguirre, A. K., Helleranta, J., Rahman, S., \& Bergman, S. (2009). Monitoring performance of electric utilities: indicators and benchmarking in subSaharan Africa. World Bank Other Operational Studies 13030, The World Bank.

Treasury, U. S. (2009). Financial Regulatory Reform: A New Foundation: Rebuilding Financial Supervision and Regulation. US White Paper on Financial Regulatory Reform.

Wymeersch, E. (2007). The structure of financial supervision in Europe: about single financial supervisors, Twin Peaks and multiple financial supervisors. European Business Organization Law Review, 8(2), 237-306. doi: https://doi.org/10.1017/S1566752907002376

\section{AGRADECIMENTO}

Agradecemos à Light S.A. pelo financiamento do Projeto de P\&D que permitiu o desenvolvimento desta pesquisa. 